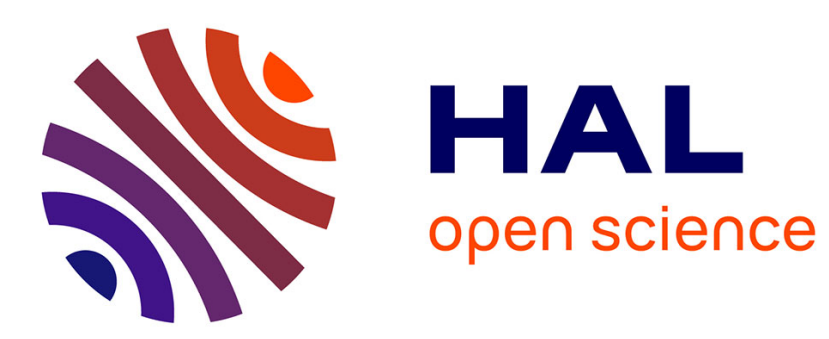

\title{
Observations relatives à la couronne visible actuellement autour du soleil
}

\author{
A. Cornu
}

\section{To cite this version:}

A. Cornu. Observations relatives à la couronne visible actuellement autour du soleil. J. Phys. Theor. Appl., 1885, 4 (1), pp.53-59. 10.1051/jphystap:01885004005300 . jpa-00238594

\section{HAL Id: jpa-00238594 https://hal.science/jpa-00238594}

Submitted on 1 Jan 1885

HAL is a multi-disciplinary open access archive for the deposit and dissemination of scientific research documents, whether they are published or not. The documents may come from teaching and research institutions in France or abroad, or from public or private research centers.
L'archive ouverte pluridisciplinaire HAL, est destinée au dépôt et à la diffusion de documents scientifiques de niveau recherche, publiés ou non, émanant des établissements d'enseignement et de recherche français ou étrangers, des laboratoires publics ou privés. 


\section{OBSERVATIONS RELATIVES A LA GOORONNE VISIBLE AGTUELLEMENT AUTOUR DU SOLEIL;}

PAR M. A. CORNU.

Le phénomène d'illumination colorée qu'on aperçoit autour du Soleil, depuis plusieurs mois, présente une foule de particularités intéressantes, qui ne me paraissent pas avoir encore été signalées; en voici une description succincte :

I $^{\circ}$ Variation de forme et de coloration. - La couronne rougeâtre, concentrique au Soleil, observée par M. Thollon (Comptes rendus des 24 mars et 8 septembre r 884 ) et par M. le $\mathrm{D}^{\mathrm{r}}$ Forel ( 1 a aût et I $^{\text {er }}$ septembre I 884) est la manifestation la plus simple de ce phénomène; elle n'apparaît, sous celte forme géométrique, que quand le Soleil est à une grande hauteur au-dessus de l'horizon, au milieu d'un ciel suffisamment pur; mais, lorsque le Soleil s'abaisse, l'arc inférieur de la couronne s'élargit et devient plus intense; au-dessous, sur l'horizon, naît une bande colorée de même teinte, qui s'élargit aussi vers la couronne, comme attirée par elle; cette bande s'etend peu à peu dans tous les sens, atteint la couronne et finit en quelque sorte par l'englober.

Pendant cette déformation, l'espace brillant intérieur, d'un blanc légèrement bleuâtre, reste sensiblement circulaire; par une illusion facile à expliquer, le Soleil paraît n'en plus occuper le centre, mais un point de plus en plus rapproché du bord inférieur; finalement, lorsque le Soleil atteint l'horizon, le cercle intérieur de la couronne y devient aussi tangent au même point (1). Dans l'intervalle, les teintes se modifient d'une manière continue; elles passent d'abord par la couleur dite jaune de Naples et tournent ensuite au jaune brunâtre. Après le coucher du Soleil, la couronne prend l'apparence d'une fumée légère et s'efface peu à peu; les colorations se fondent avec celles du Soleil couchant, mais en rap-

( $\left.{ }^{1}\right)$ J'ai observé, pour la première fois, cette apparence à la fin du mois de novembre dernier, le lendemain de la première apparition des beaux crépuscules: depuis, je l'ai revue à chaque coucher de soleil dans un ciel pur.

J. de Phys., $2^{\circ}$ série, t. IV. (Février 1885.) 
pelant le plus souvent, dans les régions supérieures, les teintes rosées des beaux crépuscules de l'hiver dernier.

Telle est la succession des apparences qu'on peut voir pendant une belle journée, lorsque le ciel est pur ; de petits nuages n'empêchent cependant pas l'observation; ils offrent même l'occasion de constater que l'illumination colorée se produit derrière eux, c'est-à-dire au-dessus d'eux, car ils se détachent en blanc bleuâtre sur les teintes rousses, orangées ou jaunes, dont il est ici question.

La brume ou les cirrhus légers suffisent généralement à effacer tous ces phénomènes; mais les cumulus épais, avec trouées de ciel bleu, permettent, dans certaines circonstances météorologiques, de faire des observations encore plus curieuses, lorsque ces trouées s'ouvrent sur la couronne. La couleur rousse assez effacée que la couronne présente d'ordinaire se change en un rouge de cuivre extrêmement vif, se fondant par diverses nuances intermédiaires avec le bleu azur sur lequel il se détache. La succession des teintes de la couronne, qu'on aperçoit mal sur le ciel pur, se voit alors avec netteté dans l'ordre suivant : à partir du centre, bleu azur clair, gris teinte neutre, jaune brun, jaune orangé, rouge cuivre, rouge pourpre et violet sombre; elle offre beaucoup d'analogie avec la succession des teintes du premier anneau des couronnes de diffraction que présentent les nuages légers : on peut donc faire souvent la comparaison directe.

L'apparition de ces teintes si vives ne se produit pas à toutes les trouées ouvertes sur la couronne; une seconde condition est nécessaire: il faut que, sur la ligne de visée, les couches brumeuses soient dans l'ombre; autrement, les couleurs sont larées de blanc et ne diffèrent en rien des teintes ordinaires; cette condition devient évidente, lorsqu'on suit les traînées blanches ou rayons qui divergent du Soleil à travers ces trouées, car c'est en dehors de ces rayons qu'on observe ces teintes rouges de feu d'un éclat si extraordinaire.

Ces observations conduisent à penser que le phénomène se produit dans une région de l'atmosphère notablement plus élevée que le niveau des cumulus et même des cirrhus ( ${ }^{1}$ ) et qu'il acquerrait,

( ${ }^{1}$ On voit, en effet, assez souvent, des nuages offrant le phénomìne du halo 
pour un observateur situé dans les régions de l'atmosphère où toule brume terrestre disparaît, l'intensité extraordinaire que j’ai entrevue dans les circonstances précitées.

C'est justement la conclusion à laquelle arrive M. le $\mathrm{D}^{\mathbf{r}}$ Forel, d'après ses observations à des altitudes croissantes dans les Alpes: cette concordance, obtenue par deux voies si différentes, donne une grande probabilité à l'hypothèse qui placerait le siège du phénomène à une altitude considérable.

$2^{\circ}$ Procédé pour augmenter la visibilité du phénomène. Les circonstances météorologiques permettant d'observer ces couleurs éclatantes sont très rares: dans nos climats du centre de la France, les teintes de la couronne sont pâles, même dans les belles journées; on peut toutefois leur donner beaucoup d'éclat par un artifice très simple; il consiste à cacher le disque du Soleil par un écran opaque et à observer le ciel à travers un milieu coloré convenable: certains verres d'un rose violacé, diverses substances en solution, parmi lesquelles on peut citer l'alun de chrome el surtout le violet d'aniline ( ${ }^{1}$ ), permettent d'apercevoir la couronne, lors même que la brume l'efface presque entièrement à l'œil nu.

Lorsqu'on a réglé la teinte des verres ou la concentration de la liqueur d'après la meilleure visibilité du phénomène, on reconnaît avec un spectroscope que l'absorbant a enlevé le milieu du spectrc (le jaune et le vert), sans avoir sensiblement altéré les couleurs extrêmes (le rouge, le bleu et le violet). Aussi la couronne apparaît-elle en rouge sur fond bleu violacé.

Il paraît nécessaire, pour une bonne visibilité, de conserver à l'absorbant la transparence pour les couleurs extrêmes; car un verre rouge à vitrail, qui éteint tout, sauf le rouge, dessine mal la couronne, et une cuve de bleu céleste (sulfate de cuivre ammoniacal), qui intercepte le rouge, le jaune et le vert, efface entière-

de $22^{\circ}$ se détacher en blanc clair sur la teinte cuivrée de la couronne : la couronne n'a comme diamètre moyen que les $\frac{2}{3}$ du diamètre du halo.

( ${ }^{1}$ ) On improvise la petite cuve nécessaire à l'observation, en collant à chaud avec de la paraffine un anneau de carton de Bristol entre deux verres. On commence par regarder le cicl à $90^{\circ}$ du Soleil pour effacer certaines impressions physiologiques persistantes, puis on approche progressivement du centre du phćnomène. 
ment le phénomène ( 1 ); le ciel paraît d'un bleu presque uniforme jusqu'au bord du disque solaire.

Il résulte de ces observations que le phénomène décroît en intensité avec la réfrangibilité de la lumière émise : nous retrouverons plus loin des conclusions semblables.

$3^{\circ}$ Observations polariscopiques. - Cette couronne circomsolaire n'a pas seulement troublé l'uniformité de la teinte bleue dı ciel autour du Soleil, elle a apporté une perturbation considérable dans la polarisation atmosphérique, spécialement aus environs des points neutres.

On sait qu'il existe dans le ciel pur (que j'appellerai normal par opposition à l'état actuel) trois points neutres, c'est-à-dire trois points dont la lumière est dépourvue de toute polarisation; à savoir : le point d'Arago, situé au-dessus du point antisolaire; le point de Babinet, au-dessus du Soleil; le point de Brewster, audessous; tous trois dans le vertical de l'astre.

Depuis l'apparition de la couronne, les distances respectives de ces trois points aux centres solaire et antisolaire ont varié d'une quantité considérable: en outre, quatre nouveaux points neutres ont apparu, deux à deux symétriquement placés par. rapport au vertical du Soleil, à peu près à la hauteur des centres solaire et antisolaire.

L'observation des deux nouveaux points neutres à droile et à gauche du Soleil est particulièrement facile en interposant un verre rouge entre l'œil et le polariscope ( $\left.{ }^{2}\right)$ de Savart; ils sont situés à l'extérieur de la couronne rouge : un verre de couleur verte montre des points neutres moins écartés, et un verre bleu cobalt les rapproche à une distance très faible du Soleil. L'intensité de

(') La photographie ne m'a donné aucunc trace appréciable de la couronnc un jour où le phénomène était passablement visible : le collodion employé avait été choisi de manière à présenter le maximum de sensibilité entre les raies $\mathrm{G}$ et $\mathrm{H}$.

$\left({ }^{2}\right)$ Il est nécessaire, pour une bonne perception des franges, de disposer le polariscope comme la loupe des horlogers, pour protéger latéralement l'œil qui observe, de couvrir l'autre œil avec la main et de maintenir tout le visage à l'ombre derrière un écran convenable. Un point ncutre est caractérisé par les deux conditions suivantes : c'est un point où les franges du polariscope s'effacent : $r^{\circ}$ quelle que soit leur orientation $; 2^{\circ}$ en devenant complémentaires sur leur prolongeinent. 
la perturbation décroît donc avec la réfrangibilité de la lumière émise: l'analyse du phénomène polariscopique, comparé à celui des années antérieures, montre que la perturbation correspond en chaque point à la superposition d'un faisceau polarisé dans un plan perpendiculaire au plan passant par le Soleil.

Les deux nouveaux points neutres, voisins du point antisolaire, sont au contraire difficiles à observer: il faut employer un double verre rouge à vitrail pour opérer sur une radiation aussi monochromatique et aussi peu réfrangible que possible. Le champ de vision est sombre : néanmoins les franges sont bien visibles si le ciel est pur; malheureusement le phénomène ne dure que quelques minutes avant le coucher du Soleil. On reconnaît alors, dans l'allure des franges observées, sous toutes les orientations, entre l'horizon et le point neutre d'Arago, l'analogue de ce qui se produit entre le Soleil et le point neutre de Babinet : on est ainsi conduit à l'existence de deux autres points neutres. Toutefois, comme l'identité n'est pas et ne peut pas être absolue, en raison de la différence des circonstances, je ferai quelques réserves sur la constitution de ces points ( 1 ) et je demanderai que l'on considère l'affirmation de leur existence comme la manière la plus simple et la plus brève de caractériser la perturbation qui existe actuellement au voisinage du point antisolaire.

4" Observations polarimétriques et photométriques. - La perturbation n'est pas moins grande aux environs du point où la polarisation est maximum, c'est-à-dire dans le vertical du Soleil à $9^{0}$ de l'astre : la proportion maximum de lumière polarisée, qui, dans les belles journées atteignait, ces années dernières, le chiffre de $o,-5$, n'a presque jamais dépassé o, 48 .

Une diminution accidentelle de la proportion de lumière polarisée correspond d'ordinaire à une simple augmentation de la brume; mais la permanence d'une proportion aussi faible, dans les conditions météorologiques les plus diverses, rapprochée des per-

( ${ }^{1}$ L'effacement des franges est à peu près complet en ces points; mais leur inversion, qui doit se faire ici sur une zone étroite, n'est pas observable avec une lumière si faible et si peu homogène. 
turbations signalées plus haut, paraît indiquer l'existence permanente d'une cause agissant comme la brume pour diffuser de la lumière neutre (ou polarisée normalement au plan du Soleil) el diminuer la polarisation apparente du ciel bleu.

Des mesures photométriques, faites autour du disque solaire, mais dont le détail m'entraînerait trop loin, conduisent à la même conclusion.

L'observation polarimétrique faite avec un verre rouge ou un verre bleu cobalt montre que la proportion de lumière polarisée est moindre pour les radiations rouges que pour les radiations bleues : là encore l'intensité de la perturbation décroîtrait donc avec la réfrangibilité.

Cette description sommaire est trop incomplète pour permettre des conclusions rigoureuses sur la cause du phénomène : elle suffit cependant à montrer que cette cause est liée vraisemblablement à l'explosion du Krakatau. Les observations rapportées plus haut seraient assez nombreuses pour appuyer l'hypothèse très naturelle d'un nuage de particules ténues d'un diamètre moyen presque constant, lancées par le volcan dans les hautes régions de l'atmosphère.

On peut en effet produire, à l'aide de corpuscules présentant des diamètres sensiblement égaux, un phénomène de diffraction qui offre avec la couronne solaire l'analogie la plus complète : l'expérience est particulièrement facile à réaliser avec les spores $\mathrm{d}$ (' champignons (lycopode, charbon de maïs, lycoperdon); il suffit d'en saupoudrer une lame de glace et d'observer, à travers le nuage ainsi formé, la flamme d'une bougie placée dans une salle obscure. Les couronnes de diffraction qu'on aperçoit autour de la lumière reproduisent les teintes du phénomène solaire, d'autant mieux que les couronnes sont plus grandes.

Au lieu de spores on peut employer des globules sanguins dilués dans une solution de sulfate de soude au $\frac{1}{20}$, qu'on emprisonne entre deux verres comme pour les observations microscopiques: une goutte de sang suffit pour faire l'expérience ( $\left.{ }^{1}\right)$. Ce phénomènc

( $\left.{ }^{1}\right)$ Ces expériences ont été projetées devant la Société de Physique par M. Jules Duboscq avec lobligeant concours de M. Max. Cornu, professeur au Muséum, à qui je dois les spores de champignons, et de M. le $D^{\mathrm{r}}$ Gréhant, aide- 
MALLARD ET LE CHATELIER. - MÉlANGES GAZEUX EXPLOSIFS. 59 des couronnes est même tellement facile à reproduire qu'on l'aperçoit partout où des grains isolés se déposent; le voile des épreuves photographiques, les buées, fumées, ou poussières qui s'attachent aux vitres, permettent toujours d'observer, par transparence, une couronne rousse qui borde le champ de lumière diffusée par les particules.

Bien que cette analogie des couronnes de diffraction avec le phénomène actuellement visible autour du Soleil donne une explication très plausible de la cause de ces apparences, je crois qu'on ne doit accepter cette explication qu'avec certaines réserves: en effet, le phénomène paraît plus complexe si l'on a égard en même temps au caractère particulier de la perturbation que présente la polarisation atmosphérique, caractère signalé plus haut, et qui tendrait à le rapprocher du halo.

Je remettrai à une époque ultérieure la discussion des causes probables de ce phénomène, lorsque les déterminations numériques que j’ai entreprises à ce sujet seront plus avancées. 